\title{
Commentary on Efficacy and Safety of Trabectedin in Metastatic Uterine Leiomyosarcoma: A Retrospective Multicenter Study of the Spanish Ovarian Cancer Research Group (GEICO)
}

\author{
María Jesús Rubio* \\ Department of Oncology, Hospital Universitario Reina Sofía, Córdoba, Spain
}

\begin{abstract}
Uterine Leiomyosarcoma (uLMS) is a very rare and aggressive carcinoma which, at advanced or recurrent stage, has a median overall survival of below 12 months. The Spanish ovarian cancer research group (GEICO), carried out a retrospective, observational, multicenter study involving 36 adult patients with unrespectable advanced or metastatic uLMS who received trabectedin after an anthracycline-containing regimen. The efficacy and safety observations found with trabectedin in this study were in line with previously shown in clinical trials.

Trabectedin provides long-term carcinoma stabilization and adequate tolerability, thus it represents an appropriate option for the treatment of advanced uLMS. Beneficial effects are optimized when administered as second-line, after the failure of the anthracycline-containing regimen, which allows achieving longer clinical benefit and disease control. Furthermore, it is recommended to maintain the treatment with trabectedin until disease progression due to its better efficacy outcomes as well as its safety profile, allowing the long-term administration with no cumulative toxicities. Trabectedin has also demonstrated activity in diverse soft tissue sarcoma subtypes.

Keywords:Uterine leiomyosarcoma; Trabectedin; Efficacy; Safety; GEICO
\end{abstract}

\section{INTRODUCTION}

Uterine Leiomyosarcoma (uLMS) is a very rare and aggressive carcinoma whose incidence is $0.36-0.64$ per 100,000 women per year [1]. The 5 -year survival varies from $16 \%$ to $57 \%$, depending the stage of the disease when diagnosed [2]. At advanced or recurrent stage, the median overall survival (OS) is below 12 months [3]. Surgical resection represents the only treatment option with the chance for cure so far, and probably a longer response than medical management [4]. Besides this, the recurrence risk within 2 and 5 years from the surgery is 40\%-70\% [5].

Doxorubicin, ifosfamide, or gemcitabine in monotherapy are some of the therapeutic armamentarium used for the treatment of uLMS, achieving objective response rates (ORR) of $13 \%-$ $25 \%, 17 \%$, and $21 \%$, respectively [6,7]. The combination of gemcitabine plus docetaxel has also been shown a response rate varying from $27 \%$ to $53 \%[8,9]$. Trabectedin is indicated for patients with advanced soft tissue sarcoma (STS) after failure of a previous treatment with anthracycline and ifosfamide, or when the patients unsuited [10]. Trabectedin received approval in 2007 by the European Medicines Agency, and in 2015 by the U.S. Food and Drug Administration, based on the efficacy and manageable safety profile demonstrated in diverse clinical trials [11,12]. Besides randomized, controlled clinical trials are the basis for the medical evidence, their applicability and generalizability require validation in non-interventional, realworld studies [13].

\section{STUDY OBSERVATIONS}

The Spanish ovarian cancer research group (GEICO), carried out a retrospective, observational, multicenter study involving 36 adult patients with unresectable advanced or metastatic uLMS who received trabectedin after an anthracycline-containing regimen [14]. The objective of the study was to determine efficacy and safety of trabectedin in routine clinical practice. The primary endpoint included its efficacy in terms of progressionfree survival (PFS), whereas secondary endpoints encompassed

Correspondence to: Dr. María Jesús Rubio, Department of oncology, Hospital Universitario Reina Sofía, Córdoba, Spain, Phone Number:+34 957 0100 00; E-mail: mjesusrubio63@gmail.com

Received: January 18, 2021; Accepted: February 03, 2021; Published: February 10, 2021

Citation: Rubio MJ (2021) Commentary on Efficacy and Safety of Trabectedin in Metastatic Uterine Leiomyosarcoma: A Retrospective Multicenter Study of the Spanish Ovarian Cancer Research Group (GEICO). Clinics Mother Child Health. 18:383

Copyright: ( (2021) Rubio MJ, et al. This is an open-access article distributed under the terms of the Creative Commons Attribution License, which permits unrestricted use, distribution, and reproduction in any medium, provided the original author and source are credited. 
OS, tumor response to trabectedin and the safety profile. The median number of trabectedin cycles was 6 (3-25 cycles). Since starting treatment with trabectedin, median PFS and OS were 5.4 and 18.5 months, respectively. The survival results of trabectedin treatment are shown in Table 1 . The ORR was $27.8 \%$, with median response duration of 11 months.

\begin{tabular}{lll}
\hline $\begin{array}{l}\text { Mean months (95\% } \\
\text { confidence interval) }\end{array}$ & $\begin{array}{l}\text { Progression-free } \\
\text { survival }\end{array}$ & Overall survival \\
\hline Total patients; $\mathrm{n}=36$ & $5.4(3.5-7.3)$ & $18.5(11.5-25.6)$ \\
\hline Regarding previous lines & \\
\hline $\begin{array}{l}\text { First/second-lines; } \\
\mathrm{n}=15\end{array}$ & $5.4(0.0-11.1)$ & $25.3(0.0-66.7)^{*}$ \\
\hline $\begin{array}{l}\text { Third line/beyond; } 5.7(3.8-7.7) \\
\mathrm{n}=21\end{array}$ & $15.1(10.9-19.2)$ \\
\hline
\end{tabular}

ECOG at trabectedin initiation

\begin{tabular}{lll}
\hline $0-1 ; n=28$ & $5.4(3.7-7.0)$ & $19.8(12.9-26.7)$ \\
\hline $2-3 ; n=4$ & $3.1(1.7-4.5)$ & $6.0(2.4-9.6)^{* *}$ \\
\hline
\end{tabular}

Overall survival statistically different between: * first/second-lines versus third line/beyond $(\mathrm{p}=0.028) ;{ }^{* *}$ ECOG $0-1$ versus $2-3(\mathrm{p}=0.013)$

Table 1: Survival results with trabectedin treatment.

Patients receiving trabectedin as first or second line (after neoadjuvant or first-line anthracycline-containing regimen), showed a significantly higher median OS (25.3 months; 95\% confidence interval, 95\%CI: $0.0-66.7)$ than those in third-line or beyond (15.1 months; 95\%CI: 10.9-19.2; $\mathrm{p}=0.028$ ). Moreover, the number of patients achieving a complete or partial response was higher when receiving trabectedin in firstline after an anthracycline-containing regimen $(16.7 \%$ and $50.0 \%$, respectively) than those in second-line or beyond $(0.0 \%$ and $20.0 \%$ ).

At trabectedin initiation, patients with Eastern Cooperative Oncology Group performance status (ECOG) $\leq 1$ also had a significantly higher median OS (19.8 months) than those with 2-3 (6.0 months; $\mathrm{p}=0.013)$. Regarding safety, most frequent adverse events were neutropenia (30.5\% of patients), anemia (25.0\%), fatigue (25.0\%), nausea (13.9\%), and vomiting $(11.1 \%)$. No cumulative toxicity was reported.

\section{CONCLUSION}

Efficacy and safety observations found with trabectedin in this study were in line with those previously shown in clinical trials [13]. Trabectedin provides long-term carcinoma stabilization and adequate tolerability, thus it represents an appropriate option for the treatment of advanced uLMS [14]. Beneficial effects are optimized when administered as second-line, after the failure of anthracycline-containing regimen, which allows achieving longer clinical benefit and disease control [13].
Furthermore, it is recommended to maintain the treatment with trabectedin until disease progression due to its better efficacy outcomes as well as its safety profile, allowing the long-term administration with no cumulative toxicities [13-15]. Trabectedin has also demonstrated activity in diverse STS subtypes, including non-uterine leiomyosarcoma and liposarcoma, as well as rare subtypes, such as synovial sarcoma, myxofibrosarcoma, malignant peripheral nerve sheath tumors, rhabdomyosarcoma, or endometrial stromal sarcoma [16].

\section{REFERENCES}

1. Skorstad M, Kent A, Lieng M. Uterine leiomyosarcoma-incidence, treatment, and the impact of morcellation. A nationwide cohort study. Acta Obstet Gynecol Scand. 2016;95(9):984-990.

2. Kapp DS, Shin JY, Chan JK. Prognostic factors and survival in 1396 patients with uterine leiomyosarcomas: Emphasis on impact of lymphadenectomy and oophorectomy. Cancer. 2008;112(4):820-830.

3. Zivanovic O, Leitao MM, Iasonos A, Jacks LM, Zhou Q. Stage-specific outcomes of patients with uterine leiomyosarcoma: A comparison of the International Federation of Gynecology and Obstetrics and American Joint Committee on Cancer Staging Systems. J Clin Oncol. 2009;27(12):2066-2072.

4. Korets SB, Curtin JP. Surgical options for recurrent uterine sarcomas. Am Soc Clin Oncol Educ Book. 2012:362-366.

5. Arend RC, Toboni MD, Montgomery AM, Burger RA, Olawaiye AB. Systemic treatment of metastatic/recurrent uterine leiomyosarcoma: A changing paradigm. Oncologist. 2018;23(12):1533-1545.

6. Look KY, Sandler A, Blessing JA, Lucci JA 3rd, Rose PG. Phase II trial of gemcitabine as second-line chemotherapy of uterine leiomyosarcoma: A Gynecologic Oncology Group (GOG) Study. Gynecol Oncol. 2004;92(2):644-647.

7. Amant F, Lorusso D, Mustea A, Duffaud F, Pautier P. Management strategies in advanced uterine leiomyosarcoma: Focus on trabectedin. Sarcoma. 2015;15(6):704-724.

8. Hensley ML, Blessing JA, Degeest K, Abulafia O, Rose PG. Fixed-dose rate gemcitabine plus docetaxel as second-line therapy for metastatic uterine leiomyosarcoma: A Gynecologic Oncology Group phase II study. Gynecol Oncol. 2008;109(3):323-328.

9. Hensley ML, Blessing JA, Mannel R, Rose PG. Fixed-dose rate gemcitabine plus docetaxel as first-line therapy for metastatic uterine leiomyosarcoma: A Gynecologic Oncology Group phase II trial. Gynecol Oncol. 2008;109(3):329-334.

10. Dubois EA, Cohen AF. Trabectedin. Br J Clin Pharmacol. 2009;68(3): 320-321.

11. Monk BJ, Dalton H, Benjamin I, Tanović A. Trabectedin as a new chemotherapy option in the treatment of relapsed platinum sensitive ovarian cancer. Curr Pharm Des. 2012;18(25):3754-3769.

12. Barone A, Chi DC, Theoret MR, Chen H, He K. FDA Approval summary: Trabectedin for unresectable or metastatic liposarcoma or leiomyosarcoma following an anthracycline-containing regimen. Clin Cancer Res. 2017;23(24):7448-7453.

13. de Sande González LM, Martin-Broto J, Kasper B, Blay JY, Le Cesne A. Real-world evidence of the efficacy and tolerability of trabectedin in patients with advanced soft-tissue sarcoma. Expert Rev Anticancer Ther. 2020;20(11):957-963.

14. Rubio MJ, Lecumberri MJ, Varela S, Alarcón J, Ortega ME. Efficacy and safety of trabectedin in metastatic uterine leiomyosarcoma: A retrospective multicenter study of the Spanish ovarian cancer research group (GEICO). Gynecol Oncol Rep. 2020;33(63):1005-10012.

15. Cesne LA, Blay JY, Domont J, Tresch-Bruneel E, Chevreau C, Bertucci $\mathrm{F}$, et al. Interruption versus continuation of trabectedin in patients 
with soft-tissue sarcoma (T-DIS): A randomised phase 2 trial. Lancet Oncol. 2015;16(3):312-319.

16. Le Cesne A, Ray-Coquard I, Duffaud F, Chevreau C, Penel N, Blay JY, et al. Trabectedin in patients with advanced soft tissue sarcoma: A retrospective national analysis of the French Sarcoma Group. Eur J Cancer. 2015;51(6):742-750 